\title{
The Virial Equation of State of Low-Density Neutron Matter
}

\author{
C.J. Horowitz ${ }^{*}$ and A. Schwenk \\ Nuclear Theory Center and Department of Physics, Indiana University, Bloomington, IN 47408
}

\begin{abstract}
We present a model-independent description of low-density neutron matter based on the virial expansion. The virial equation of state provides a benchmark for all nuclear equations of state at densities and temperatures where the interparticle separation is large compared to the thermal wavelength. We calculate the second virial coefficient directly from the nucleon-nucleon scattering phase shifts. Our results for the pressure, energy, entropy and the free energy correctly include the physics of the large neutron-neutron scattering length. We find that, as in the universal regime, thermodynamic properties of neutron matter scale over a wide range of temperatures, but with a significantly reduced interaction coefficient compared to the unitary limit.
\end{abstract}

PACS numbers: 21.65.+f, 26.50.+x, 97.60.Bw, 05.70.Ce

Keywords: Low-density neutron matter, equation of state, virial expansion

\section{INTRODUCTION}

Matter composed of neutrons and protons exhibits remarkable properties. Cold neutron matter is close to a scaling regime for densities $n \lesssim 1 / 10 n_{0}$, where $n_{0}=0.16 \mathrm{fm}^{-3}$ denotes nuclear saturation density. In this regime, thermodynamic properties of neutron matter scale only with the density, and for the energy per particle $E / N$ one has approximately [1, 2]

$$
\frac{E}{N} \approx 0.5\left(\frac{E}{N}\right)_{\text {free }}=0.5 \frac{3\left(3 \pi^{2}\right)^{2 / 3}}{10 m} n^{2 / 3}
$$

where $m$ is the nucleon mass. In contrast, below saturation density, the energy per particle of symmetric nuclear matter is independent of the density $E / A \approx-16 \mathrm{MeV}$, and nucleons cluster into nuclei and larger structures. The physics of nuclear matter is therefore a crossover from a gas of nucleons to homogeneous matter, where nuclei and larger clusters coexist with the nucleon gas over a wide range of intermediate densities. Nuclear clustering is due to the competition of nuclear binding, entropy and the Coulomb repulsion.

With this rich interplay, it is very important to develop a reliable formalism for nucleonic matter over densities and temperatures that are experimentally or observationally accessible. In this paper, we present a model-independent description of neutron matter based on the virial expansion. We have previously applied the virial expansion to low-density nuclear matter composed of neutrons, protons and alpha particles [3]. The virial equation of state presents a controlled application of nucleon scattering data to densities and temperatures where the interparticle separation is large compared to the thermal wavelength. For neutron matter, the relevant densities are $n \lesssim 4 \cdot 10^{11}(T / \mathrm{MeV})^{3 / 2} \mathrm{~g} / \mathrm{cm}^{3}$, where $T$ is the temperature. The virial equation of state therefore pro-

\footnotetext{
*E-mail: horowit@indiana.edu

†E-mail: schwenk@indiana.edu
}

vides important constraints on the physics of the neutrinosphere in supernovae, where one has $T \approx 4 \mathrm{MeV}$ and $n \sim 10^{11} \mathrm{~g} / \mathrm{cm}^{3} \sim 1 / 1000 n_{0}$ [4, 5].

Properties of dilute Fermi gases can also be studied in laboratory experiments with trapped atoms. Cold atom experiments provide exciting access to neutron matter in the universal low-density regime, where the scattering length $a_{s}$ is large compared to the interparticle separation $k_{\mathrm{F}} a_{s} \gg 1$ and the effective range is small $k_{\mathrm{F}} r_{e} \ll 1$. Under these conditions, there are no length scales associated with the interaction, and the only dimensionful scale is the Fermi momentum $k_{\mathrm{F}}$. Therefore, the system will exhibit universal behavior, where all macroscopic observables are given by powers of $k_{\mathrm{F}}$ multiplied by universal factors. For example, under these conditions the energy per particle $E / N$ for cold gases of atomic ${ }^{6} \mathrm{Li},{ }^{40} \mathrm{~K}$ or neutrons with equal populations of two spin states is

$$
\frac{E}{N}=\xi\left(\frac{E}{N}\right)_{\text {free }}=\xi \frac{3 k_{\mathrm{F}}^{2}}{10 m}
$$

where the universal factor $\xi$ is a number. For neutrons, the scattering length is unnaturally large with $a_{n n}=-18.5 \pm 0.3 \mathrm{fm}$ (for a recent review see [6]). The effective range is expected to be approximately chargeindependent, and thus $r_{n n}=2.7 \mathrm{fm} \mathrm{[]}$. Consequently, the universal regime is restricted to extremely low neutron densities $n=k_{\mathrm{F}}^{3} /\left(3 \pi^{2}\right)<10^{-4} \mathrm{fm}^{-3}$.

Dilute Fermi gases with resonant interactions were realized for the first time by O'Hara et al. in 2002 8]. In this and subsequent experiments, the universal factor $\xi$ was determined by extracting an equation of state from the properties of the atomic cloud [8, 9, 10, 11]. This leads to $\xi=0.51 \pm 0.04[9], \xi \approx 0.7[10]$ and $\xi=$ $0.27_{-0.09}^{+0.12}[1]$, for temperatures in units of the Fermi temperature $T / T_{\mathrm{F}} \approx 0.05$ (except for $T / T_{\mathrm{F}} \approx 0.6$ in $[10]$ ). To date, the most reliable theoretical results for the universal equation of state are from $T=0$, fixed-node Green's function Monte Carlo simulations, $\xi=0.44 \pm 0.01$ [12] and $\xi=0.42 \pm 0.01[13$ ].

The virial expansion has been applied to cold atoms in the vicinity of Feshbach resonances by Ho et al. [14, 15]. 
The resulting universal equation of state for high temperatures describes many observed properties of resonant Fermi gases, with a universal virial coefficient that is independent of temperature. In this paper, we study to what extent the virial equation of state of low-density neutron matter approaches this unitary limit.

There are many theoretical approaches to low-density neutron matter. Recently, the equation of state of neutron matter at zero temperature has been calculated using an effective field theory for large scattering length and large effective range [2]. This provides a modelindependent description of neutron matter for densities where the interparticle separation is comparable to the effective range. The effective field theory calculation is particularly transparent, since the couplings are fitted directly to the scattering length and the effective range.

In addition, there are microscopic calculations starting from nucleon-nucleon (NN) and three-nucleon interactions that reproduce NN scattering and selected fewnucleon data. One of these are Hartree-Fock calculations [16] with the model-independent low-momentum interaction $V_{\text {low } k}$ [17]. These results are promising, because low-momentum interactions offer the possibility of a perturbative and thus systematic approach to nucleonic matter [18]. Moreover, for neutron matter at subnuclear densities, the Hartree-Fock results agree with complicated Fermi hyper-netted chain [19, 20] and Brueckner 21, 22] calculations. However, these approaches may not be reliable for very low densities, where the large scattering length physics is important. This is especially problematic for Brueckner calculations, since the holeline expansion relies on Pauli blocking effects being dominant, so that all large structures are dissolved in the medium. Note that the equation of state calculation of Buchler and Coon [23] is close in strategy to the virial equation of state, but it takes into account Pauli blocking on the phase shifts and thus uses a model NN interaction. There are also a number of Skyrme-type [24, 25, 26] and relativistic mean-field [27, 28, 29] parametrizations of the nuclear energy functional, which are used to calculate ground-state energies and densities of intermediate-mass and heavy nuclei. However, the resulting energy functional for neutron matter is not well constrained from fits to finite nuclei.

Finally, there are promising lattice simulations for neutron matter using effective field theory, where the couplings are regularized on the same lattice [30]. These calculations are presently limited to small lattices and to the lowest-order contact interaction, which is fitted to reproduce the scattering length. In this paper, we study how the virial equation of state depends on NN scattering properties and in particular on the physics beyond the large scattering length. As a result, we assess how a low-order truncation in the effective field theory impacts the equation of state of low-density neutron matter. This provides a valuable check for the lattice results.

The virial equation of state is a general, modelindependent equation of state for a dilute gas, provided the fugacity $z=e^{\mu / T}$ is small. Here $\mu$ denotes the chemical potential. An additional assumption is that the system is in a gas phase and has undergone no phase transition with decreasing temperature or increasing density. Under these conditions, the partition function can be expanded in powers of the fugacity. The second virial coefficient $b_{2}$ describes the $z^{2}$ term in this expansion and is directly related to the two-body scattering phase shifts 31, 32. Little is known about the third virial coefficient, which describes the $z^{3}$ term [33, 34]. We emphasize that the virial expansion is not a perturbative expansion in powers of $k_{\mathrm{F}} a_{s}$. A great advantage of the virial formalism is that it includes both bound states and scattering resonances on an equal footing. It correctly predicts that thermodynamic quantities, such as the pressure, are continuous as the interaction is changed to convert a low-energy scattering resonance into a weaklybound state. This continuity has been demonstrated experimentally with cold atoms in the crossover region of a Feshbach resonance (see e.g., [10]) and was also shown theoretically by Ho and Mueller using the viral expansion across $a_{s}= \pm \infty$ 14.

This paper is organized as follows. We briefly introduce the virial equation of state in Section [II Further details can be found in [3]. In Section [II] we present results for the second virial coefficient, the pressure, energy, entropy and the free energy of low-density neutron matter. We also study the dependence of the virial coefficient on NN scattering properties and show that neutron matter scales to a very good approximation. Finally, we conclude in Section IV

\section{VIRIAL EQUATION OF STATE}

For the virial equation of state we expand the grandcanonical partition function or the pressure in a power series of the fugacity

$$
P=\frac{2 T}{\lambda^{3}}\left(z+z^{2} b_{n}+z^{3} b_{n}^{(3)}+\mathcal{O}\left(z^{4}\right)\right),
$$

where $\lambda$ denotes the nucleon thermal wavelength $\lambda=$ $(2 \pi / m T)^{1 / 2}$ and $b_{n}, b_{n}^{(3)}$ are the second and third virial coefficients for neutron matter respectively. We will include $b_{n}^{(3)}$ only to make an error estimate for our virial results. The density follows from differentiating the pressure with respect to the fugacity $n=z / T\left(\partial_{z} P\right)_{V, T}$ and is given by

$$
n=\frac{2}{\lambda^{3}}\left(z+2 z^{2} b_{n}+3 z^{3} b_{n}^{(3)}+\mathcal{O}\left(z^{4}\right)\right) .
$$

Therefore, the fugacity expansion is an expansion in powers of $n \lambda^{3}$, and for finite temperatures, it is valid to much higher densities than the $k_{\mathrm{F}} a_{s}$ expansion. In this work, we truncate the virial expansion after second order in the fugacity. This leads to an equation of state that is thermodynamically consistent. 
The dependence of the density on $z$ can be inverted. This gives the virial equation of state directly in terms of density and temperature $P=P(z(n, T), T)$. In practice, we directly, without this inversion, generate the equation of state in tabular form for a range of fugacity values. This maintains the thermodynamic consistency of the virial equation of state.

The second virial coefficient is related to the partition function of the two-particle system $\sum_{\text {states }} e^{-E_{2} / T}$, where the sum is over all two-particle states of energy $E_{2}$. This sum can be converted to an integral over relative momentum $k$ weighted by the density of states of the interacting two-particle system. The density of states, and therefore the second virial coefficient, can then be expressed in terms of the scattering phase shift $\delta(k)$, summed over all allowed partial waves [31, 32].

For neutron matter, the second virial coefficient $b_{n}$ is given by

$$
b_{n}(T)=\frac{1}{2^{1 / 2} \pi T} \int_{0}^{\infty} d E e^{-E / 2 T} \delta^{\text {tot }}(E)-2^{-5 / 2},
$$

where $-2^{-5 / 2}$ is the free Fermi gas contribution and $\delta^{\text {tot }}(E)$ is the sum of the isospin-triplet elastic scattering phase shifts at laboratory energy $E$. This sum is over all partial waves with two-particle spin $S$, angular momentum $L$ and total angular momentum $J$ allowed by spin statistics, and includes a degeneracy factor $(2 J+1)$,

$$
\begin{aligned}
\delta^{\mathrm{tot}}(E) & =\sum_{S, L, J}(2 J+1) \delta_{2 S+1} \mathrm{~L}_{J}(E) \\
& =\delta^{1} \mathrm{~S}_{0}+\delta^{3} \mathrm{P}_{0}+3 \delta^{3} \mathrm{P}_{1}+5 \delta^{3} \mathrm{P}_{2}+5 \delta^{1} \mathrm{D}_{2}+\ldots
\end{aligned}
$$

Note that we have neglected the effects of the mixing parameters due to the tensor force. We expect that their contributions to the second virial coefficient describing spin-averaged observables vanish.

Finally, the entropy $S$ and the energy $E$ are obtained from the virial equation of state using thermodynamics [14, 32]. The entropy density $s=S / V$ follows from differentiating the pressure with respect to the temperature $s=\left(\partial_{T} P\right)_{\mu}$. This leads to

$$
s=\frac{5 P}{2 T}-n \log z+\frac{2 T}{\lambda^{3}} z^{2} b_{n}^{\prime},
$$

where $b_{n}^{\prime}(T)=d b_{n}(T) / d T$ denotes the temperature derivative of the virial coefficient. The energy density $\epsilon=E / V$ can be calculated from the entropy density,

$$
\epsilon=T s+n \mu-P=\frac{3}{2} P-\frac{2 T^{2}}{\lambda^{3}} z^{2} b_{n}^{\prime} .
$$

For completeness, the entropy per particle $S / N$, energy per particle $E / N$ and the free energy per particle $F / N$ are given by

$$
\frac{S}{N}=\frac{s}{n}, \quad \frac{E}{N}=\frac{\epsilon}{n} \quad \text { and } \quad \frac{F}{N}=\frac{f}{n},
$$

with the free energy density $f=\epsilon-T s$.

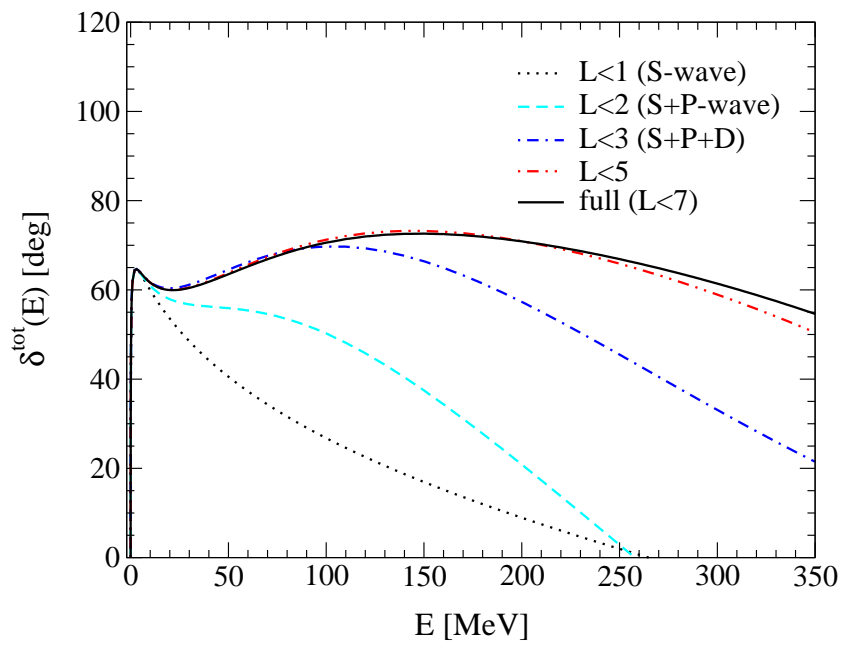

FIG. 1: (Color online) The total phase shift $\delta^{\text {tot }}(E)$ versus laboratory energy $E$ obtained from including successively higher partial waves.

\section{RESULTS}

\section{A. Virial Coefficients}

We first calculate the second virial coefficient $b_{n}$. We use the $T=1 \mathrm{np}$ phase shifts obtained from the Nijmegen partial wave analysis 35. This neglects the small charge dependences in nuclear interactions. We have included all partial waves with $L \leqslant 6$ and the resulting total total phase shift $\delta^{\text {tot }}(E)$ is shown in Fig. 11. The total phase shift displays both the prominent low-energy ${ }^{1} \mathrm{~S}_{0}$ resonance and the significance of higher partial waves. We observe that $\delta^{\text {tot }}(E)$ is very weakly dependent on energy with $\delta^{\text {tot }} \approx 65$ degrees over the entire range $E \leqslant 350 \mathrm{MeV}$ of the Nijmegen partial wave analysis. As shown in Fig. 1. the decrease of the ${ }^{1} \mathrm{~S}_{0}$ phase shift due to the large effective range is compensated by the contributions from higher angular momenta. For reference, in the unitary limit of a S-wave resonance $\left(a_{s}= \pm \infty\right)$, the phase shift is energy-independent with $\delta_{1} \mathrm{~S}_{0}=90$ degrees.

In the unitary limit, the second virial coefficient is independent of the temperature and given by $b_{2}=3 / 2^{5 / 2}=$ 0.53 [14]. For neutron matter, our results for the virial coefficient $b_{n}$ and its derivative $T b_{n}^{\prime}(T)$ are listed in Table In addition to the full results, we also give the virial coefficient obtained only from the S-wave scattering length $a_{n p}$ and including the effective range contribution. As expected, $b_{n}$ is dominated by the large S-wave scattering length, but effective range and higher partial wave contributions are significant even for these low temperatures. As a result of the weak energy dependence of $\delta^{\text {tot }}(E)$, we find that the second virial coefficient is approximately independent of temperature over a wide range, and consequently $T b_{n}^{\prime} \approx 0$. The value we obtain for $b_{n} \approx 0.31$ is $40 \%$ reduced compared to the universal value $b_{2}=0.53$. 
TABLE I: The second virial coefficient $b_{n}$ for different temperatures. In addition to the full results, we also give results for the virial coefficient calculated in the unitary limit and from including only the S-wave scattering length $a_{n p}$, with and without the effective range $r_{e}$ contribution $\left(a_{n p}=-23.768 \mathrm{fm}\right.$ and $\left.r_{e}=2.68 \mathrm{fm}[7]\right)$. The results labeled CIB take into account the effects due to charge-independence breaking (CIB) on the scattering length with $a_{n n}=-18.5 \mathrm{fm}$.

\begin{tabular}{|c|c|c|c|c|c|c|c|}
\hline$T[\mathrm{MeV}]$ & $a_{s}= \pm \infty$ & $a_{s}= \pm \infty, r_{e}$ & $a_{n p}$ & $a_{n p}, r_{e}$ & $b_{n}$ full & $b_{n}$ full with CIB & $T b_{n}^{\prime}$ full \\
\hline 1 & 0.530 & 0.449 & 0.357 & 0.287 & 0.288 & 0.251 & 0.032 \\
\hline 2 & 0.530 & 0.417 & 0.400 & 0.298 & 0.303 & 0.273 & 0.012 \\
\hline 3 & 0.530 & 0.394 & 0.421 & 0.296 & 0.306 & 0.279 & 0.004 \\
\hline 4 & 0.530 & 0.376 & 0.434 & 0.291 & 0.306 & 0.283 & 0.001 \\
\hline 5 & 0.530 & 0.360 & 0.443 & 0.285 & 0.306 & 0.285 & 0.000 \\
\hline 6 & 0.530 & 0.347 & 0.450 & 0.278 & 0.306 & 0.286 & 0.001 \\
\hline 7 & 0.530 & 0.335 & 0.456 & 0.272 & 0.307 & 0.288 & 0.002 \\
\hline 8 & 0.530 & 0.324 & 0.460 & 0.265 & 0.307 & 0.289 & 0.004 \\
\hline 9 & 0.530 & 0.314 & 0.464 & 0.259 & 0.308 & 0.291 & 0.007 \\
\hline 10 & 0.530 & 0.305 & 0.467 & 0.254 & 0.309 & 0.292 & 0.009 \\
\hline 12 & 0.530 & 0.289 & 0.472 & 0.243 & 0.310 & 0.295 & 0.013 \\
\hline 14 & 0.530 & 0.275 & 0.476 & 0.233 & 0.313 & 0.299 & 0.017 \\
\hline 16 & 0.530 & 0.263 & 0.479 & 0.224 & 0.315 & 0.302 & 0.020 \\
\hline 18 & 0.530 & 0.252 & 0.482 & 0.216 & 0.318 & 0.305 & 0.022 \\
\hline 20 & 0.530 & 0.242 & 0.484 & 0.208 & 0.320 & 0.308 & 0.023 \\
\hline 22 & 0.530 & 0.233 & 0.486 & 0.201 & 0.322 & 0.311 & 0.023 \\
\hline 24 & 0.530 & 0.224 & 0.488 & 0.194 & 0.324 & 0.313 & 0.022 \\
\hline
\end{tabular}

TABLE II: The second virial coefficient $b_{n}$ for temperatures $T \geqslant 25 \mathrm{MeV}$. As discussed in the text, we estimate an error of $<5 \%$ for these higher temperatures due to the truncation of the integration over the phase shifts at $E \leqslant 350 \mathrm{MeV}$.

\begin{tabular}{c|cc}
\hline \hline$T[\mathrm{MeV}]$ & $b_{n}$ full & $T b_{n}^{\prime}$ full \\
\hline 25 & 0.325 & 0.022 \\
30 & 0.329 & 0.015 \\
35 & 0.330 & 0.004 \\
40 & 0.330 & -0.009 \\
45 & 0.328 & -0.025 \\
50 & 0.324 & -0.041 \\
\hline \hline
\end{tabular}

In Table [ we also study the effects of chargeindependence breaking (CIB) on the scattering length. Due to the lack of neutron-neutron scattering data, we estimate CIB effects by subtracting the virial coefficient calculated only with $a_{n p}$ from the full $b_{n}$, and then add the virial coefficient obtained from the neutron-neutron scattering length $a_{n n}=-18.5 \mathrm{fm}$. We find that CIB effects are largest for $T<5 \mathrm{MeV}$ and lead to a $10 \%$ reduction of the second virial coefficient.

Finally, we extend our results for $b_{n}$ to temperatures $T \geqslant 25 \mathrm{MeV}$ in Table We give these results separately, because there is a small error for these higher temperatures due to the truncation of the integration over the phase shifts at $E \leqslant 350 \mathrm{MeV}$ (the extent of the partial wave analysis). Assuming the total phase shift is constant, we vary the energy cutoff to $E>350 \mathrm{MeV}$ and estimate the error to be $<5 \%$ up to the highest temperatures given in Table

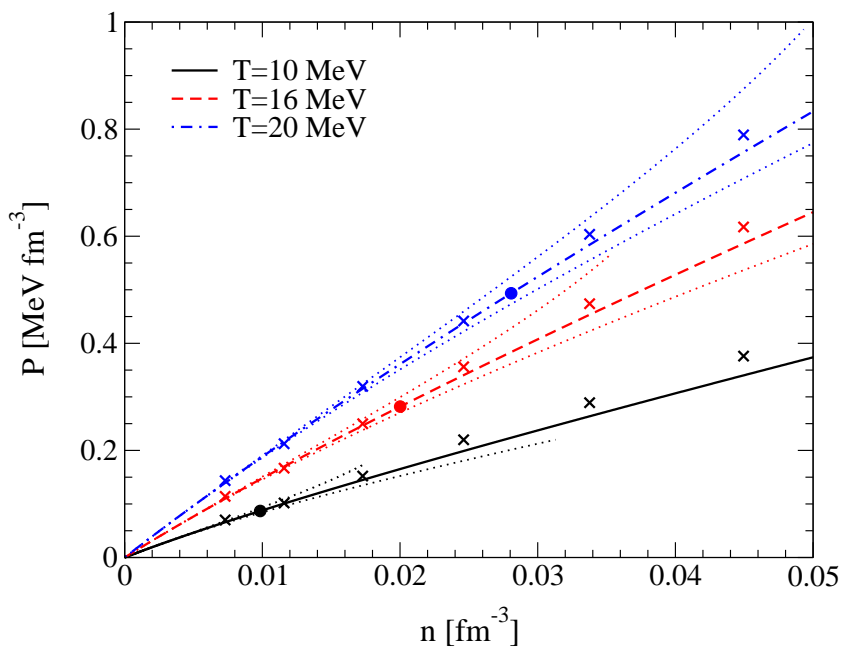

FIG. 2: (Color online) The pressure $P$ versus density $n$ for $T=10,16$ and $20 \mathrm{MeV}$. The dotted error bands for the virial equation of state are based on an estimate of a neglected third virial coefficient $b_{n}^{(3)}= \pm b_{n} / 2$. Note that a negative $b_{n}^{(3)}$ increases the pressure. Also shown are the FHNC results of Friedman and Pandharipande (crosses) [19]. The circles indicate where the fugacity is $z=0.5$, and the error bands are shown for $z \leqslant 1$. For this density range, the fugacities are $z<1.65,1.01$ and 0.79 for $T=10,16$ and $20 \mathrm{MeV}$.

\section{B. Pressure}

Our virial results for the pressure are shown in Fig. 2 for temperatures $T=10,16$ and $20 \mathrm{MeV}$. The third virial coefficient can be used to make a simple error es- 


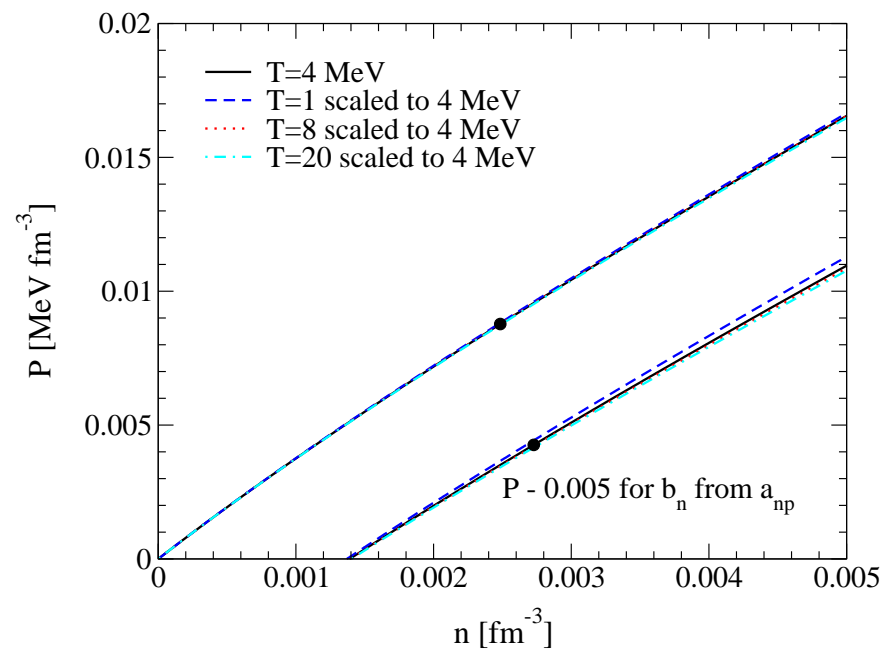

FIG. 3: (Color online) The pressure $P$ versus density $n$ calculated for $T=1,4,8$ and $20 \mathrm{MeV}$ and scaled to a temperature of $T^{\prime}=4 \mathrm{MeV}$ (for details see text). The lower curves are results for the scaled pressures obtained with a virial coefficient calculated only from the S-wave scattering length $a_{n p}$. The latter curves have been shifted for better readability. The circles indicate where the fugacity is $z=0.5$, and for this density range, the fugacity is $z<0.87$.

timate of neglected terms in the virial expansion. The Pauli principle prevents three neutrons to interact in the S-wave and there is no three-neutron bound state. Moreover, $3 \mathrm{~N}$ forces are very small in low-density neutron matter. Therefore, we consider a third virial coefficient $\left|b_{n}^{(3)}\right| \lesssim b_{n} / 2$ reasonable. Note that for the ideal Fermi gas $b_{n}^{(3)}=3^{-5 / 2}=0.06$. In Fig. 2] we show the resulting error bands, which are small even up to $z \leqslant 1$. We also compare our results to the the microscopic Fermi hypernetted-chain (FHNC) equation of state of Friedman and Pandharipande [19]. We find that the FHNC results are in very good agreement with the model-independent virial equation of state. Moreover, it is intriguing that the FHNC results lie within the error band for the virial pressure even for fugacities $z>0.5$. In contrast, we have found a disagreement of the FHNC results with the virial equation of state for low-density nuclear matter due to clustering [3].

\section{Scaling and Energy}

If the virial coefficients are temperature independent, then the power series in the fugacity for the pressure and the density will have no explicit temperature dependence. Consequently, the dependence of the pressure on density and temperature will be given by

$$
P(n, T)=T^{5 / 2} f\left(n / T^{3 / 2}\right),
$$

where $f(x)$ is a scaling function of $n / T^{3 / 2}$. If this scaling relation holds, one can predict the pressure $P\left(n^{\prime}, T^{\prime}\right)$ at

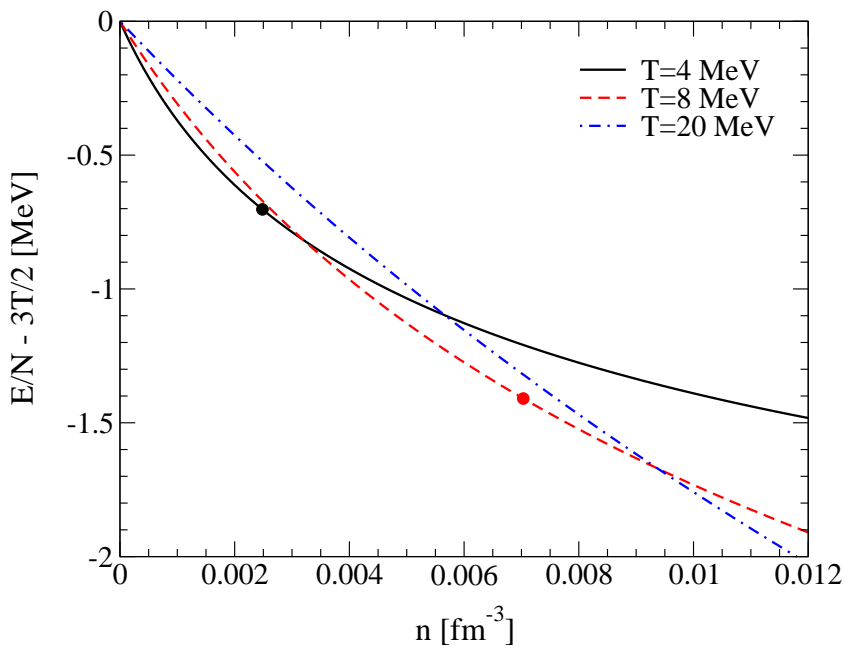

FIG. 4: (Color online) The energy per particle $E / N$ versus density $n$ for $T=4,8$ and $20 \mathrm{MeV}$. In order to clearly show the interaction effects we have subtracted the free kinetic energy $3 T / 2$ from all curves. The energy per particle obtained using the universal relation $\epsilon=3 P / 2$ with the corresponding virial pressures are indistinguishable from these curves. The circles indicate where the fugacity is $z=0.5$, and for this density range, the fugacities are $z<1.60,0.77$ and 0.25 for $T=4,8$ and $20 \mathrm{MeV}$.

a new temperature $T^{\prime}$ from $P(n, T)$ through

$$
P\left(n^{\prime}, T^{\prime}\right)=\left(\frac{T^{\prime}}{T}\right)^{5 / 2} P(n, T),
$$

with $n^{\prime}=\left(T^{\prime} / T\right)^{3 / 2} n$. In Fig. 3 we demonstrate that low-density neutron matter scales according to Eq. (10). The predicted pressures for $T^{\prime}=4 \mathrm{MeV}$ obtained from virial pressures calculated for $T=1,8$ and $20 \mathrm{MeV}$ are in excellent agreement with the unscaled $T=4 \mathrm{MeV}$ virial pressure. Fig. 3 also gives results for the scaled pressures ( $T=1,8$ and $20 \mathrm{MeV}$ scaled to $T^{\prime}=4 \mathrm{MeV}$ and unscaled $T=4 \mathrm{MeV}$ ) obtained from the virial equation of state with $b_{n}$ calculated only from the S-wave scattering length $a_{n p}$. Although Table ! shows that $b_{n}$ increases by $35 \%$ from $T=1$ to $20 \mathrm{MeV}$ in this case, the scaling symmetry continues to hold to a good approximation.

In the scaling regime, $b_{n}^{\prime}=0$ and therefore the energy density, Eq. (8), is given by

$$
\epsilon=3 P / 2 .
$$

This is a general thermodynamic relation in the universal regime 36]. In Fig. 4] we show the energy per particle for $T=4,8$ and $20 \mathrm{MeV}$. In order to separate the interaction effects we have subtracted the free kinetic energy $3 T / 2$ from all curves. We have also calculated the energy per particle from the virial pressures using the universal relation Eq. (11). The resulting curves for the energy per particle are indistinguishable from those shown in Fig. [4 This demonstrates that the $b_{n}^{\prime}$ term is indeed negligible. 


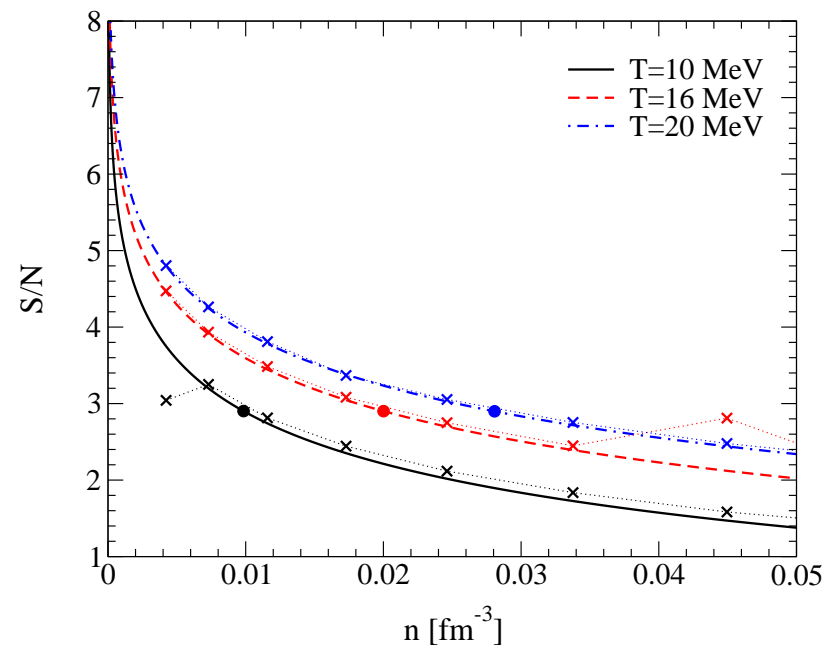

FIG. 5: (Color online) The entropy per particle $S / N$ versus density $n$ for $T=10,16$ and $20 \mathrm{MeV}$. Also shown are the FHNC results of Friedman and Pandharipande (crosses) 19]. The circles indicate where the fugacity is $z=0.5$.

While neutron matter scales as in the unitary limit, the thermodynamic properties depend on the value of the virial coefficient, and the latter depends on the physics of NN scattering.

Finally, we emphasize that the universal relation for the energy density, Eq. (11), holds independent of the value of the universal interaction coefficient given by $\xi$ for $T=0$ and $b_{n}$ for higher temperatures. In fact, Eq. (11) is valid for $T=0$, where the energy per particle only scales with density, $E / N \sim \xi n^{2 / 3}$ according to Eq. (2), and for the virial regime, where the energy per particle scales with density and temperature $E / N \sim \xi\left(T / n^{2 / 3}\right) n^{2 / 3} \sim \widetilde{\xi}\left(T / n^{2 / 3}\right) T$, with Fermi temperature $T_{\mathrm{F}} \sim n^{2 / 3}$. The latter scaling is very explicit to lowest order in the density, where the energy per particle is given by

$$
\frac{E}{N} \approx \frac{3}{2} T\left(1-b_{n} \lambda^{3} n / 2\right)
$$

\section{Entropy and Free Energy}

We present our virial results for the entropy per par-

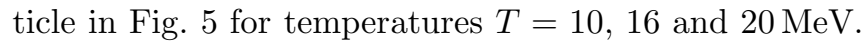
The FHNC results are again in very good agreement with the model-independent virial equation of state, with the exception of two FHNC points that seem to either reflect numerical instabilities or are typos in [19]. As indicated by the circles for $z=0.5$ in Fig. [5 we observe that the range of validity of the virial equation of state is bounded by a constant entropy per particle, independent of the temperature. This is easily understood from the expression for the entropy per particle in the scaling regime

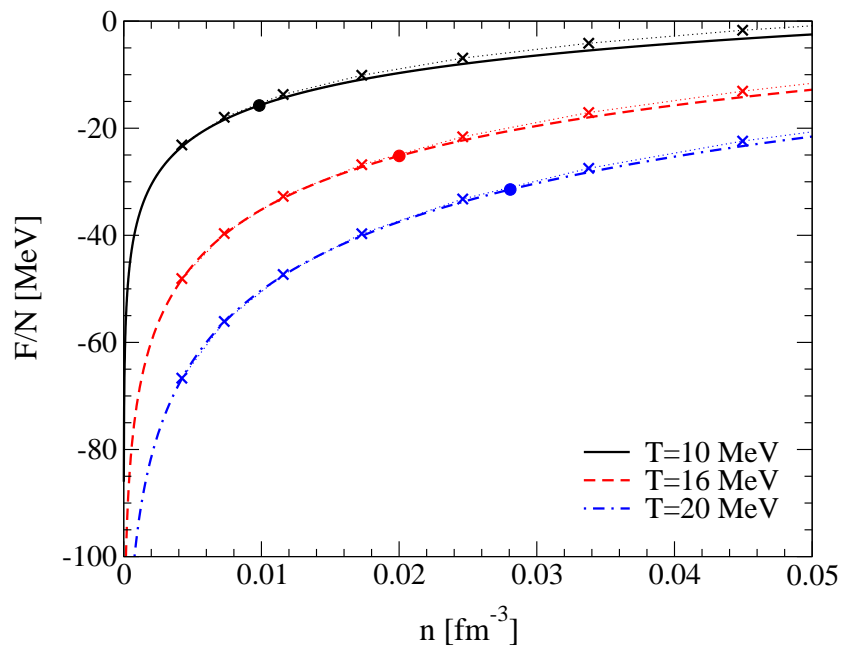

FIG. 6: (Color online) The free energy per particle $F / N$ versus density $n$ for $T=10,16$ and $20 \mathrm{MeV}$. Also shown are the FHNC results of Friedman and Pandharipande (crosses) [19]. The circles indicate where the fugacity is $z=0.5$.

with $b_{n}^{\prime}=0$,

$$
\frac{S}{N}=\frac{5}{2}\left(1-\frac{z b_{n}}{1+2 z b_{n}}\right)-\log z
$$

Therefore, if one takes as the range of validity of the virial equation of state $z \lesssim 0.5$, this is equivalent to $S / N \gtrsim 2.9$ for $b_{n}=0.31$, in agreement with Fig. 5 For completeness, we also show the free energy per particle in Fig. 6 As for the pressure and the entropy, the FHNC results agree well with our model-independent virial predictions.

\section{CONCLUSIONS}

We have presented the virial equation of state of lowdensity neutron matter. The virial expansion provides a systematic way to include strong interactions with a well-defined range of validity. The resulting virial equation of state has a simple parametric form and is thermodynamically consistent. We have calculated the second virial coefficient directly from the NN scattering phase shifts. Therefore, the virial equation of state is modelindependent and sets a benchmark for all nuclear equations of state at low densities.

We have found that the second virial coefficient $b_{n}$ is approximately temperature-independent. This is the result of an intriguing cancellation. The decrease of the ${ }^{1} \mathrm{~S}_{0}$ phase shift due to the effective range is compensated by the increase of the higher angular momentum phase shifts. Therefore, while $b_{n}$ is generally dominated by the low-energy ${ }^{1} \mathrm{~S}_{0}$ resonance, contributions from higher partial waves are significant even for these low temperatures. The virial coefficient $b_{n} \approx 0.31$ is reduced by $40 \%$ compared to the unitary limit, where $b_{2}=0.53$. 
The virial equation of state was used to make modelindependent predictions for the pressure, energy, entropy and the free energy of low-density neutron matter over a wide range of densities and temperatures. Our results include the physics of the large neutron scattering length in a tractable way. The range of validity of the virial equation of state is given by the fugacity. With $z \lesssim 0.5$, one has for the entropy per particle $S / N \gtrsim 2.9$ or for the density $n \lesssim 4 \cdot 10^{11}(T / \mathrm{MeV})^{3 / 2} \mathrm{~g} / \mathrm{cm}^{3}$. The virial expansion thus provides important constraints on the physics of the neutrinosphere in supernovae.

We have made simple error estimates of the virial equation of state by studying the effects of a neglected third virial coefficient $\left|b_{n}^{(3)}\right| \lesssim b_{n} / 2$. The resulting error bands are small. For a better error estimate, it is important to have a reliable calculation of the third virial coefficient. The FHNC results of Friedman and Pandharipande [19] are in very good agreement with the model-independent virial equation of state. This is in contrast to our findings for low-density nuclear matter [3]. The FHNC and virial results agree within the estimated errors even for densities, where $z>0.5$.

The temperature independence of $b_{n}$ leads to a scaling symmetry of low-density neutron matter. As in the universal regime, thermodynamic properties of neutron matter scale over a wide range of temperatures. This extends the approximate scaling of the $T=0$ equation of state according to Eq. (11) to finite temperatures. Finally, model-independent predictions for the density and spin response of low-density neutron matter and a detailed comparison with nuclear lattice calculations will be discussed in future work.

\section{Acknowledgments}

This work is supported by the US Department of Energy under Grant No. DE-FG02-87ER40365 and the National Science Foundation under Grant No. PHY0244822 .
[1] J. Carlson, J. Morales, V.R. Pandharipande and D.G. Ravenhall, Phys. Rev. C68 (2003) 025802.

[2] A. Schwenk and C.J. Pethick, Phys. Rev. Lett. 95 (2005) 160401.

[3] C.J. Horowitz and A. Schwenk, nucl-th/0507033

[4] M. Costantinit, A. Ianni and F. Visanni, Phys. Rev. D70 (2004) 043006.

[5] C. Lunardini and A.Y. Smirnov, Astropart. Phys. 21 (2004) 703.

[6] A. Gardestig and D.R. Phillips, Phys. Rev. C73 (2006) 014002.

[7] The neutron-proton effective range parameters are wellconstrained from the Nijmegen PWA93: $a_{\mathrm{np}}=-23.768 \pm$ $0.006 \mathrm{fm}$ and $r_{\mathrm{np}}=2.68 \pm 0.01 \mathrm{fm}$ (M.C.M. Rentmeester, private communication).

[8] K.M. O'Hara, S.L. Hemmer, M.E. Gehm, S.R. Granade and J.E. Thomas, Science 298 (2002) 2179.

[9] M.E. Gehm, S.L. Hemmer, S.R. Granade, K.M. O'Hara and J.E. Thomas, Phys. Rev. A68 (2003) 011401(R); J. Kinast, A. Turlapov, J.E. Thomas, Q. Chen, J. Stajic and K. Levin, Science 307 (2005) 1296.

[10] T. Bourdel, J. Cubizolles, L. Khaykovich, K.M.F. Magalhaes, S. Kokkelmans, G.V. Shlyapnikov and C. Salomon, Phys. Rev. Lett. 91 (2003) 020402.

[11] M. Bartenstein, A. Altmeyer, S. Riedl, S. Jochim, C. Chin, J.H. Denschlag and R. Grimm, Phys. Rev. Lett. 92 (2004) 120401 and cond-mat/0412712

[12] J. Carlson, S.-Y. Chang, V.R. Pandharipande and K.E. Schmidt, Phys. Rev. Lett. 91 (2003) 050401; S.Y. Chang, J. Carlson, V.R. Pandharipande and K.E. Schmidt, Phys. Rev. A70 (2004) 043602.

[13] G.E. Astrakharchik, J. Boronat, J. Casulleras and S. Giorgini, Phys. Rev. Lett. 93 (2004) 200404.

[14] T.-L. Ho and E.J. Mueller, Phys. Rev. Lett. 92 (2004) 160404.

[15] T.-L. Ho and N. Zahariev, cond-mat/0408469

[16] A. Schwenk, B. Friman and G.E. Brown, Nucl. Phys.
A713, 191 (2003); see also Fig. 1 in A. Schwenk, nucl-th/0411070

[17] S.K. Bogner, T.T.S. Kuo and A. Schwenk, Phys. Rept. 386 (2003) 1.

[18] S.K. Bogner, A. Schwenk, R.J. Furnstahl and A. Nogga, Nucl. Phys. A763 (2005) 59.

[19] B. Friedman and V.R. Pandharipande, Nucl. Phys. A361 (1981) 502.

[20] A. Akmal, V.R. Pandharipande and D.G. Ravenhall, Phys. Rev. C58 (1998) 1804.

[21] G. Bao, L. Engvik, M. Hjorth-Jensen, E. Osnes and E. Østgaard, Nucl. Phys. A575 (1994) 707.

[22] M. Baldo, in Nuclear Methods and the Nuclear Equation of State, International Review of Nuclear Physics, Vol. 8, World Scientific, 1999.

[23] J.-R. Buchler and S.A. Coon, Astrophys. J. 212 (1977) 807.

[24] S. Goriely, M. Samyn, P.-H. Heenen, J. M. Pearson and F. Tondeur, Phys. Rev. C66 (2002) 024326.

[25] M. Bender, P.-H. Heenen and P.-G. Reinhard, Rev. Mod. Phys. 75 (2003) 121.

[26] M.V. Stoitsov, J. Dobaczewski, W. Nazarewicz, S. Pittel and D.J. Dean, Phys. Rev. C68 (2003) 054312.

[27] P. Ring, Prog. Part. Nucl. Phys. 37 (1996) 193.

[28] B.D. Serot and J.D. Walecka, Int. J. Mod. Phys. E6 (1997) 515.

[29] R.J. Furnstahl and B.D. Serot, Nucl. Phys. A671 (2000) 447.

[30] D. Lee and T. Schäfer, Phys.Rev. C72 (2005) 024006.

[31] E. Beth and G.E. Uhlenbeck, Physica 4 (1937) 915.

[32] K. Huang, Statistical Mechanics, Wiley N.Y., Second Edition (1987). For the relation of the second virial coefficient $b_{2}$ to the two-body elastic scattering phase shifts, see p. 224-227.

[33] A. Pais and G.E. Uhlenbeck, Phys. Rev. 116 (1959) 250.

[34] P.F. Bedaque and G. Rupak, Phys. Rev. B67 (2003) 174513. 
[35] V.G.J. Stoks, R.A.M. Klomp, M.C.M. Rentmeester and J.J. de Swart, Phys. Rev. C48 (1993) 792; see also NNOnLine, http://nn-online.org

[36] T.-L. Ho, Phys. Rev. Lett. 92 (2004) 090402. 